\title{
Advanced Looping Broadcast Proxy Re-Encryption in Cloud computing
}

\author{
Pavan Yadav1)
}

\begin{abstract}
Recently, various augmented Proxy Re-Encryptions (PRE), e.g. Conditional (CPRE), identity-based PRE (IPRE) and broadcast PRE (BPRE), have been proposed for adaptable applications. By joining CPRE, IPRE and BPRE, this paper proposes an adaptable primitive alluded to as conditional identity-based broadcast PRE (CIBPRE) and formalizes its semantic security. CIBPRE permits a sender to scramble a message to numerous recipients by determining these collectors' personalities, and the sender can appoint a re-encryption key to an intermediary so he can change over the underlying ciphertext into another one to another arrangement of proposed beneficiaries. Additionally, the re-encryption key can be connected with a condition to such an extent that exclusive the coordinating ciphertexts can be re-encoded, which permits the first sender to authorize get to control over his remote ciphertexts in a fine-grained way. We propose a proficient CIBPRE plot with provable security. In the instantiated scheme, the initial ciphertext, the re-encrypted ciphertext and the re-encryption key are all in consistent size, and the parameters to create a re-encryption key are autonomous of the first beneficiaries of any underlying ciphertext. At last, we demonstrate an utilization of our CIBPRE to secure cloud email system invaluable over existing secure email systems in view of Pretty Good Privacy protocol or identity-based encryption.
\end{abstract}

Keywords : proxy re-encryption, cloud storage, identity-based encryption, broadcast encryption, secure cloud email.

\section{Introduction}

Endeavors have been made to furnish PRE with adaptable abilities. The early PRE was proposed in the conventional public-key infrastructure setting which incurs complicated certificate management[1-5]. To alleviate from this issue, a few character based PRE (IPRE) plans[6-8] were proposed so that the receivers' recognizable identities can serve as open keys. Rather than bringing and checking the collectors' testaments, the sender and the intermediary simply need to know the beneficiaries' personalities, which is more helpful practically speaking.

PRE and IPRE permit a single receiver. On the off chance that there are more beneficiaries, the framework needs to summon PRE or IPRE numerous circumstances. To address this issue,

Received(December 14, 2015), Review Result(1st: December 31, 2015, 2015, 2nd: February 11, 2016), Accepted(March 10, 2016)

${ }^{1}$ (Corresponding Author) Vidyaa Vikas Educational Institutions, Mysore - Bannur Road, Alanahally, Mysuru, Karnataka, India

email: yadavpav2726@gmail.com 
the idea of communicate PRE (BPRE) has been proposed[9-11]. BPRE works correspondingly as PRE and IPRE yet more flexible. Conversely, BPRE permits a sender to produce an underlying ciphertext to a collector set, rather than a solitary beneficiary. Advance, the sender can designate a re-encryption key connected with another beneficiary set so that the proxy can re-encrypt to.

The above PRE schemes just permit the re-encryption strategy is executed in a win or bust way. The proxy can either re-scramble all the underlying ciphertexts or none of them. This coarse-picked up control over ciphertexts to be re-scrambled may restrain the use of PRE systems. To fill this crevice, a refined idea alluded to as contingent PRE (CPRE) has been proposed. In CPRE plans[6-13], a sender can authorize fine-grained re-encryption control over his underlying ciphertexts. The sender accomplishes this objective by partner a condition with a reencryption key. Just the ciphertexts meeting the predefined condition can be re-encoded by the proxy holding the corresponding re-encryption key.

\section{Proposed System}

\subsection{Related Work}

The main PRE plan was proposed by Blaze, Bleumer and Strauss in [1]. Taking after this original work, various PRE plans [19-25] have been proposed in the customary open key setting. These PRE plans require authentications to demonstrate the legitimacy of open keys. A client needs to confirm the authentications before scrambling a plaintext.

So as to maintain a strategic distance from the overhead to check open keys' declarations, a few IPRE plans [3-5] have been introduced by consolidating the possibility of character based encryption [16]. The plan in [3] is demonstrated secure in the irregular prophet (RO) display in which a hash capacity is expected completely arbitrary. Interestingly, the plan in [4] is demonstrated secure in the standard model. The plan in [5] is demonstrated secure in a more grounded security sense, i.e., vagary against picked ciphertext assault in the standard model.

The above PRE schemes just permit information partaking in a coarse-grained way. That is, if the client assigns a reencryption key to the proxy, all ciphertexts can be reencrypted and after that be available to the expected clients; else none of the ciphertexts can be re-scrambled or got to by others. This issue is tended to in the late CPRE plans [6-13] permitting finegrained information sharing. The plans in [8][12][13] are demonstrated secure against picked 
ciphertext assault. The contingent character based PRE (CIPRE) plots in [6-8] consolidates the basic thoughts of CPRE and IPRE. Essentially, the two contingent communicate PRE conspires in [9] joins the thoughts of CPRE and communicate encryption, and are secure against picked plaintext assaults and picked ciphertext assaults, separately. Notwithstanding fine-grained information sharing, an additional preferred standpoint of these CBPRE plans is that it permits one to impart information to numerous clients in a more proficient manner.

A few other discretionary properties have been accomplished in late PRE plans. The PRE plots in [13][24][25] are furnished with an additional property that the beneficiary of a ciphertext is unknown. The plans in [26-27] accomplish multi-utilize bidirectional re-encryption. A ciphertext can be re-scrambled different circumstances. Additionally, a re-encryption key understands the bidirectional share between two clients. In particular, if Alice designates a re-encryption key to an intermediary for re-encoding her ciphertexts to Bob. The re-encryption key can likewise empower to re-encode Bob's ciphertexts to Alice. These two PRE plans are provably secure under the picked ciphertext assault individually in the irregular prophet and standard models. Interestingly, the PRE scheme in [21] is multi-utilize unidirectional PRE plots in which bidirectional re-encryption is illegal. The work in [28] characterizes a general idea for PRE, which is called deterministic limited automata-based useful PRE (DFA-based FPRE), and proposes a solid DFA-based FPRE framework. The late work in [29] proposes cloud-based revocable personality based intermediary re-encryption that supports user revocation and delegation of decryption rights.

\subsection{Existing System}

Proxy Re-Encryption (PRE) gives a secure and flexible technique for a sender to store and share information. A client may scramble his record with his own public key and after that store the ciphertext in an honest-but-curious server. At the point when the beneficiary is chosen, the sender can assign a re-encryption key connected with the collector to the server as an intermediary. At that point the intermediary re-encodes the underlying ciphertext to the planned collector. At long last, the recipient can decode the subsequent ciphertext with her private key. The security of PRE for the most part guarantees that (1) neither the server/intermediary nor non-planned beneficiaries can take in any helpful data about the (re-)encrypted file, and (2) before receiving the re-encryption key, the proxy cannot re-encrypt the initial ciphertext in a meaningful way. Endeavors have been made to outfit PRE with flexible capacities. The early PRE was proposed in the customary open key framework setting 
which causes convoluted declaration administration. To assuage from this issue, a few personality based PRE (IPRE) plans were proposed so that the beneficiaries' conspicuous characters can serve as open keys. Rather than getting and checking the collectors' authentications, the sender and the intermediary simply need to know the recipients' identities, which is more convenient in practice

\subsubsection{Disadvantage of Existing System}

The early PRE was proposed in the traditional public- key infrastructure setting which incurs complicated certificate management.

\subsection{Proposed System}

In this paper, we refine PRE by incorporating the advantages of IPRE, CPRE and BPRE for more adaptable applications and propose another idea of conditional identitybased broadcast PRE (CIBPRE). In a CIBPRE system, a trusted key generation focus (KGC) introduces the system parameters of CIBPRE, and creates private keys for clients. To safely share documents to numerous beneficiaries, a sender can encode the records with the receivers' identities and file-sharing conditions. In the event that later the sender might likewise want to impart a few documents related to a similar condition with different collectors, the sender can assign a reencryption key named with the condition to the intermediary, and the parameters to produce the re-encryption key is free of the first recipients of these records. At that point the intermediary can re-scramble the underlying ciphertexts coordinating the condition to the subsequent beneficiary set. With CIBPRE, notwithstanding the underlying approved recipients who can get to the record by unscrambling the underlying ciphertext with their private keys, the recently approved beneficiaries can likewise get to the document by decoding the re-scrambled ciphertext with their private keys. Take note of that the underlying ciphertexts might be put away remotely while keeping mystery. The sender does not have to download and re-encode tediously, but rather assigns a solitary key coordinating condition to the intermediary. These features make CIBPRE a versatile tool to secure remotely stored files, especially when there are different receivers to share the files as time passes.

\subsubsection{Advantages of Proposed System}

It allows a user to share their outsourced encrypted data with others in a fine-grained 
manner. All CIBPRE users take their identities as public keys to encrypt data.

It avoids a user to fetch and verify other users' certificates before encrypting his data.

Moreover, it allows a user to generate a broadcast ciphertext for multiple receivers and share his outsourced encrypted data to multiple receivers in a batch manner.

\subsection{System Architecture}

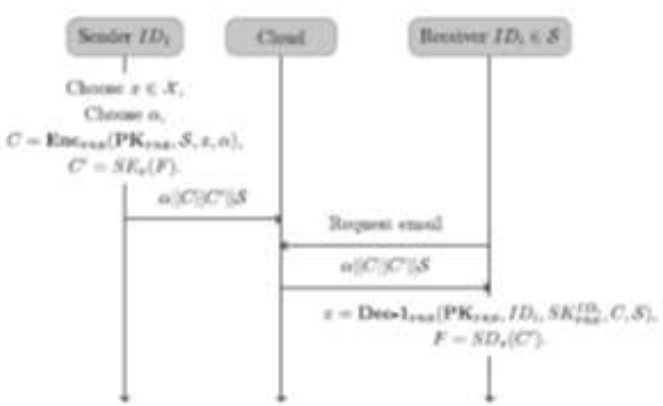

(a) Send an email.

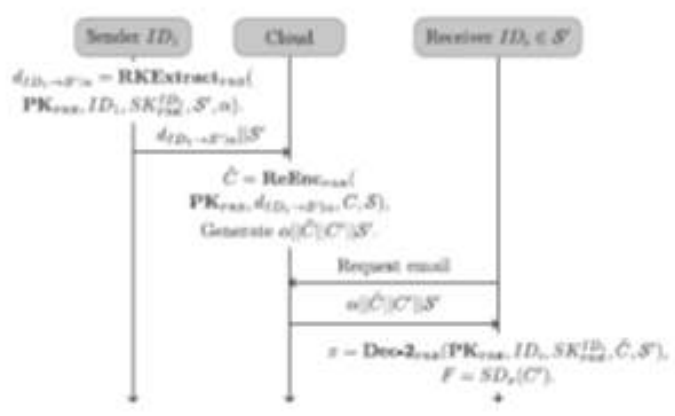

(b) Forward an email.

[Fig 1] System Architecture

\subsection{Modules}

We have two main modules,

Key Management Module

Send an Encrypted Cloud Mail Module

\subsubsection{Key Management}

In this phase, when a new user joins this system, the KGC generates a private key for him. Without loss of generality, let ID denote the email address of the new user. To generate the private key, and sends it to the user in a secure channel which is established by the SSL/TLS protocol.

\subsubsection{Send an Encrypted Cloud Email}

In this phase, a user can send an encrypted email to other users. And this email will be 
stored in the cloud server. If the user wants to review this email, he can fetch the encrypted email from the cloud server and decrypt it. Suppose user ID1 wants to send the email content $\mathrm{F}$ (including the associated attachment) to the users

\section{Conclusion}

This paper presented a new kind of PRE concept called conditional identity-based broadcast proxy re-encryption(CIBPRE), as well as its IND-sID-CPA security definitions. The CIBPRE is a general concept equipped with the capabilities of conditional PRE, Identity-based PRE and broadcast PRE. The IND-sID-CPA security definition of CIBPRE incorporated the security requirements of CPRE, IPRE and BPRE.

CIBPRE inherits the advantages of CPRE, IPRE and BPRE for applications. It allows a user to share their outsourced encrypted data with others in a fine-grained manner. All CIBPRE users take their identities as public keys to encrypt data. It avoids a user to fetch and verify other users'certificates before encrypting his data. Moreover, it allowsa user to generate a broadcast ciphertext for multiple receivers and share his outsourced encrypted data to multiple receivers in a batch manner. We instantiated the first CIBPRE scheme based on the Identity-based broadcast encryption in [30]. Upon the provable security of the IBBE scheme and the DBDH assumption, the instance of CIBPRE is provably IND-sIDCPA secure in the RO model. It indicates that without the corresponding private key or the right to share a user's outsourced data, one can learn nothing about the user's data.

Finally, we compared the proposed CIBPRE scheme with similar works and the comparison confirms the advantages of our CIBPRE scheme. We built the encrypted cloud email system based our CIBPRE scheme. Compared with the previous techniques such as PGP and IBE, our CIBPRE-based system is much more efficient in the aspect of communication and more practical in user experience.

\section{References}

[1] M. Blaze, G. Bleumer and M. Strauss, Divertible protocols andatomic proxy cryptography, Proc. Int. Conf. Theory Appl. CrytographicTechn.: Adv. Cryptol., (1998), pp.127-144.

[2] A. Boldyreva, M. Fischlin, A. Palacio and B. Warinschi, A closerlook at PKI: Security and efficiency, Proc. 10th Int. Conf. PracticeTheory Public-Key Cryptography, (2007), pp.458-475. 
[3] M. Green and G. Ateniese, Identity-based proxy re-encryption, Proc. 5th Int. Conf. Appl. Cryptography Netw. Security, (2007), pp. 288-306.

[4] T. Matsuo, Proxy re-encryption systems for identity-basedencryption," in Proc. 1st Int. Conf. Pairing-Based Cryptography, (2007), pp.247-267.

[5] C. K. Chu and W. G. Tzeng, Identity-based proxy re-encryptionwithout random oracles, Proc. 10th Int. Conf. Inf. Security, (2007), pp.189-202.

[6] L. Ibraimi, Q. Tang, P. Hartel and W. Jonker, A type-and-identity-based proxy re-encryption scheme and its application in healthcare, in Proc. 5th VLDB Conf. Secure Data Manage., (2008), pp.185-198.

[7] J. Shao, G. Wei, Y. Ling and M. Xie, Identity-based conditionalproxy re-encryption, Proc. IEEE Int. Conf. Commun., (2011), pp.1-5.

[8] K. Liang, Z. Liu, X. Tan, D. S. Wong and C. Tang, A CCA-secureidentity-based conditional proxy re-encryption without random oracles, in Proc. 15th Int. Conf. Inf. Security Cryptol., (2012), pp.231-146.

[9] C. K. Chu, J. Weng, S. S. M. Chow, J. Zhou and R. H. Deng, Conditional proxy broadcast re-encryption, Proc. 14th Australasian Conf. Inf. Security Privacy, (2009), pp.327-342.

[10] Q. Tang, Type-based proxy re-encryption and its construction, in Proc. 9th Int. Conf. Cryptol. India: Progress Cryptol., (2008), pp.130-144.

[11] J. Weng, R. H. Deng, X. Ding, C.-K. Chu and J. Lai, Conditionalproxy re-encryption secure against chosen-ciphertext attack, Proc. 4th Int. Symp. Inf., Comput. Commun.Security, (2009), pp.322-332.

[12] J. Weng, Y. Yang, Q. Tang, R. H. Deng and F. Bao, Efficient conditionalproxy re-encryption with chosen-ciphertext security, Proc. 12th Int. Conf. Inf. Security, (2009), pp.151-166.

[13] L. Fang, W. Susilo and J. Wang, Anonymous conditional proxyre-encryption without random oracle, Proc. 3rd Int. Conf. ProvableSecurity, (2009), pp.47-60.

[14] K. Liang, Q. Huang, R. Schlegel, D. S. Wong and C. Tang, A conditionalproxy broadcast re-encryption scheme supporting timed release, Proc. 9th Int. Conf. Inf. Security Practice Experience, (2013), pp.132-146.

[15] P. R. Zimmermann, PGP Source Code and Internals. Cambridge, MA, USA: MIT Press, (1995).

[16] D. Boneh and M. Franklin, Identity-based encryption from the weil pairing, Proc. 21st Annu. Int. Cryptol.: Adv. Cryptol., (2001), pp.213-239.

[17] Radicati Group, Cloud business email market, 2014-2018 [Online], (2014). Available: http://www.radicati.com/wp/wp-content/uploads/2014/10/Cloud-Business-Email-Market-2014-2018-Executive-Sum mary.pdf

[18] Proofpoint Group, Cloud-based archiving vs. on-premiseslegacy archiving [Online], (2012), Available: http://video.proofpoint.com/id/cloud-based-archiving-vs.-on-premises-legacy-archiving-TCO-white-paper

[19] G. Ateniese, K. Fu, M. Green and S. Hohenberger, Improved proxy reencryption schemes with applications to secure distributed storage, ACM Trans. Inf. Syst. Security, (2006), Vol.9, pp.1-30.

[20] R. H. Deng, J. Weng, S. Liu and K. Chen, Chosen-ciphertextsecure proxy re-encryption without pairings, Cryptol. Netw. Security, (2008), Vol.5339, pp.1-17. 
[21] V. Kirtane and C. P. Rangan, RSA-TBOS signcryption with proxyre-encryption, Proc. 8th ACM Workshop Digital Rights Manage., (2008), pp.59-66.

[22] B. Libert and D. Vergnaud, Unidirectional chosen-ciphertextsecure proxy re-encryption, Proc. 11th Int. Workshop Practice Theory, (2008), pp.360-379.

[23] J. Shao and Z. Cao, CCA-secure proxy re-encryption without pairings, Proc. 12th Int. Conf. Practice Theory Public Key Cryptography, (2009), pp.357-176.

[24] G. Ateniese, K. Benson and S. Hohenberger, Key-private proxyre-encryption, Proc. Cryptographers Track RSA Conf. TopicsCryptol., (2009), pp.279-294.

[25] J. Shao, P. Liu, G. Wei and Y. Ling, Anonymous proxy reencryption, Security Commun. Netw., (2012), Vol.5, No.5, (2012), pp.439-449.

[26] R. Canetti and S. Hohenberger, Chosen-ciphertext secure proxyreencryption, in Proc. 14th ACM Conf. Comput. Commun. Security, (2007), pp.185-194.

[27] T. Matsuda, R. Nishimaki and K. Tanaka, CCA proxy re-encryption without bilinear maps in the standard model, Proc. 13th Int. Conf. Practice Theory Public Key Cryptography, (2010), pp.261-278.

[28] K. Liang, M. H. Au, J. K. Liu, X. Qi, W. Susilo, X. P. Tran, D. S.Wong and G. Yang, A DFA-based functional proxy re-encryption scheme for secure public cloud data sharing, IEEE Trans. Inf. Forensics Security, (2014), Vol.9, No.10, pp.1667-1680.

[29] K. Liang, J. K. Liu, D. S. Wong and W. Susilo, An efficient cloud based revocable identity-based proxy re-encryption scheme for public clouds data sharing, Proc. Eur. Symp. Res. Comput. Security, (2014), pp. $257-272$.

[30] C. Delerablée, Identity-based broadcast encryption with constantsize ciphertexts and private keys, Proc. 13th Int. Conf. TheoryAppl. Cryptol. Inf. Security: Adv. Cryptol., (2007), pp.200-215. 